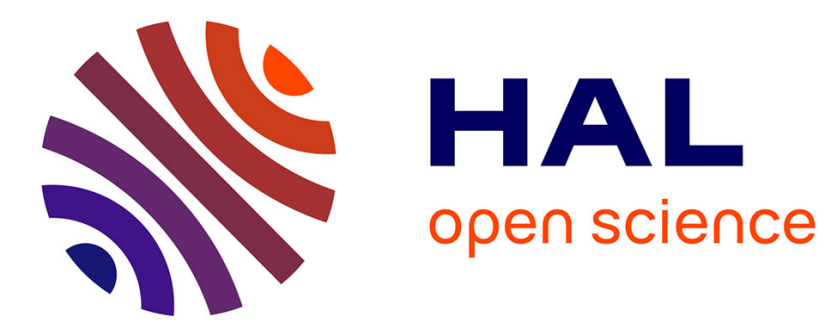

\title{
Multipoint formulas for scattered far field in multidimensions
}

Roman Novikov

\section{To cite this version:}

Roman Novikov. Multipoint formulas for scattered far field in multidimensions. Inverse Problems, 2020, 36 (9), 095001(12 pp.). 10.1088/1361-6420/aba891 . hal-02611264

\section{HAL Id: hal-02611264 \\ https://hal.science/hal-02611264}

Submitted on 18 May 2020

HAL is a multi-disciplinary open access archive for the deposit and dissemination of scientific research documents, whether they are published or not. The documents may come from teaching and research institutions in France or abroad, or from public or private research centers.
L'archive ouverte pluridisciplinaire HAL, est destinée au dépôt et à la diffusion de documents scientifiques de niveau recherche, publiés ou non, émanant des établissements d'enseignement et de recherche français ou étrangers, des laboratoires publics ou privés. 


\section{Multipoint formulas for scattered far field in multidimensions}

\section{R.G. Novikov}

CMAP, CNRS, Ecole Polytechnique, Institut Polytechnique de Paris, 91128 Palaiseau, France;

IEPT RAS, 117997 Moscow, Russia

e-mail: novikov@cmap.polytechnique.fr

Abstract. We give asymptotic formulas for finding the scattering amplitude at fixed frequency and angles (scattered far field) from the scattering wave function given at $n$ points in dimension $d \geq 2$. These formulas are explicit and their precision is proportional to $n$. To our knowledge these formulas are new already for $n \geq 2$.

Keywords: Helmholtz equation, Schrödinger equation, scattered near field, scattered far field, inverse problems

AMS subject classification: 35P25, 35R30

\section{Introduction}

We consider the scattering problem for the equation

$$
-\Delta \psi+v(x) \psi=E \psi, \quad x \in \mathbb{R}^{d}, d \geq 1, E>0,
$$

where

$$
\begin{aligned}
& v \in L^{\infty}\left(\mathbb{R}^{d}\right), \text { supp } v \subset D, \\
& D \text { is an open bounded domain in } \mathbb{R}^{d} .
\end{aligned}
$$

Equation (1.1) can be considered as the Schrödinger equation of quantum mechanics at fixed energy $E$ that describes a particle interacting with a macroscopic object contained in $D$. In this case $v$ is interpreted as the potential of this interaction.

Equation (1.1) can be also considered as the Helmholtz equation of electrodynamics or acoustics at fixed frequency $\omega$. In this case $v$ and $E$ can be interpreted as follows:

$$
v(x)=(1-(n(x)))^{2}\left(\frac{\omega}{c_{0}}\right)^{2}, \quad E=\left(\frac{\omega}{c_{0}}\right)^{2},
$$

where $n(x)$ is a scalar index of refraction, $n(x) \equiv 1$ on $\mathbb{R}^{d} \backslash D, c_{0}$ is a reference speed of wave propagation.

For equation (1.1) we consider the scattering solutions $\psi^{+}(x, k), k \in \mathbb{R}^{d}, k^{2}=E$, specified by the following asymptotics as $|x| \rightarrow \infty$ :

$$
\psi^{+}(x, k)=e^{i k x}+\frac{e^{i|k||x|}}{|x|^{(d-1) / 2}} f_{1}\left(k,|k| \frac{x}{|x|}\right)+O\left(\frac{1}{|x|^{(d+1) / 2}}\right),
$$

for some a priori unknown $f_{1}$. The function $f_{1}$ arising in (1.3) is the far field pattern, or scattering amplitude, for equation (1.1) for fixed $E$ and is defined on

$$
\mathcal{M}_{E}=\left\{k, l \in \mathbb{R}^{d}: \quad k^{2}=l^{2}=E\right\}=\mathbb{S}_{\sqrt{E}}^{d-1} \times \mathbb{S}_{\sqrt{E}}^{d-1} .
$$


Remark 1.1. For mathematical convenience in many works the terminology "scattering amplitude" is used for the function $f$ such that

$$
\begin{aligned}
& f_{1}(k, l)=c(d,|k|) f(k, l), \quad(k, l) \in \mathcal{M}_{E}, \\
& c(d,|k|)=-\pi i(-2 \pi i)^{(d-1) / 2}|k|^{(d-3) / 2} .
\end{aligned}
$$

In order to study the scattering solutions $\psi^{+}$and the scattering amplitude $f_{1}$ one can use the Lippmann-Schwinger integral equation (2.1) and the integral formulas (2.5), (2.6); see Section 2.

We consider, in particular, the following problems for equation (1.1), under assumptions (1.2):

Problem 1.1a. Reconstruct potential $v$ from its scattering amplitude $f_{1}$.

Problem 1.1b. Reconstruct potential $v$ from $\psi^{+}$appropriately given outside of $D$.

Problem 1.2. Find $f_{1}$ from $\psi^{+}$appropriately given outside of $D$.

A recent survey on Problems 1.1 (mainly, on Problem 1.1a) is given in [N3].

In the present work we are focused on Problem 1.2.

In addition, one can see that results on Problem 1.2 and Problem 1.1a admit straightforward applications to Problem 1.1b.

Note that formula (1.4), under our assumptions for $d=1$, is reduced to the formula

$$
\psi^{+}(x, k)=e^{i k x}+e^{i|k||x|} f_{1}\left(k,|k| \frac{x}{|x|}\right), \quad \text { for } \quad x \in \mathbb{R} \backslash D, \quad k \in \mathbb{R} \backslash\{0\} .
$$

One can see that formula (1.8) solves Problem 1.2 for $d=1$. However, Problem 1.2 is much more complicated for $d>1$.

The first formula for solving Problem 1.2 for $d>1$ was given in Theorem 3.3 of [Ber] for the case when $d=3$. This formula gives $f_{1}(k, l)$ at fixed $k \in \mathbb{S}_{\sqrt{E}}^{2}$ it term of an infinite sum of integrals of $\psi^{+}(x, k)-e^{i k x}$ in $x \in \partial D$ for the case when $D=B_{R}$ for some $R>0$, $d=3$, where

$$
B_{r}=\left\{x \in \mathbb{R}^{d}:|x|<r\right\}, \quad r>0 .
$$

Formula (2.6) recalled in Section 2 can be also considered as a formula for solving Problem 1.2 for $d \geq 1$.

A disadvantage of the aforementioned well-known formulas for solving Problem 1.2 for $d>1$ consists in the following: for finding $f_{1}(k, l)$ at fixed $k, l \in \mathbb{S}_{\sqrt{E}}^{d-1}$ values of $\psi^{+}(x, k)$ along the whole boundary $\partial D$ are necessary. In addition, the formula of [Ber] is complicated, whereas formula $(2.6)$ requires not only $\psi^{+}$on $\partial D$ but also its normal derivative on $\partial D$.

In the present work we give, in particular, for fixed $(k, l) \in \mathcal{M}_{E}$,

$$
\begin{aligned}
& \text { formulas for finding } f_{1}(k, l) \text { up to } O\left(s^{-n}\right) \text { as } s \rightarrow+\infty \\
& \text { from } \psi^{+}(x, k) \text { given at } n \text { points } x=x_{1}(s), \ldots, x_{n}(s)
\end{aligned}
$$

where

$$
\begin{aligned}
& x_{j}(s)=\left(s+\tau_{j}\right) \hat{l}, \quad j=1, \ldots, n, \hat{l}=l /|l|, \\
& s>0, \tau_{1}=0, \tau_{j_{1}}<\tau_{j_{2}} \text { for } j_{1}<j_{2},
\end{aligned}
$$


Multipoint formulas for scattered far field

or

$$
\begin{aligned}
& x_{j}(s)=\lambda_{j} s \hat{l}, \quad j=1, \ldots, n, \hat{l}=l /|l|, \\
& s>0, \lambda_{1}=1, \lambda_{j_{1}}<\lambda_{j_{2}} \text { for } j_{1}<j_{2} .
\end{aligned}
$$

These formulas are explicit and are presented in detail below in Introduction and in Section 3. To our knowledge these formulas are new already for $n \geq 2$; for $n=1$ these formulas are reduced to formula (1.4) considered as a formula for finding $f_{1}$ from $\psi^{+}$.

Let $z=z(x, k)$ be defined as follows:

$$
\psi^{+}(x, k)=e^{i k x}+\frac{e^{i|k||x|}}{|x|^{(d-1) / 2}} z(x, k)
$$

where $x, k \in \mathbb{R}^{d} \backslash\{0\}$.

Assume for simplicity in (1.11a) that

$$
\tau_{j}=(j-1) \tau, \quad j=1, \ldots, n, \tau>0
$$

Then our formulas (1.10), (1.11a) take the form:

$$
\begin{aligned}
& f_{1}(k, l)=f_{1, n}(k, l, \tau, s)+O\left(s^{-n}\right) \text { as } s \rightarrow+\infty \\
& f_{1, n}(k, l, \tau, s)=\frac{1}{\tau^{n-1}} \sum_{j=1}^{n} \frac{(-1)^{n-j}\left(s+\tau_{j}\right)^{n-1} z\left(\left(s+\tau_{j}\right) \hat{l}, k\right)}{(j-1) !(n-j) !}
\end{aligned}
$$

see formulas (3.7), (3.5) of Section 3.

In fact, the present work continues studies of the recent work [N2]. In particular, in [N2], for fixed $(k, l) \in \mathcal{M}_{E}, k \neq l$, for $d=3$ and $d=2$, we give

formulas for finding $f_{1}(k, l)$ up to $O\left(s^{-n}\right)$ as $s \rightarrow+\infty$

from $\left|\psi^{+}(x, k)\right|^{2}$ given at $2 n$ points $x=x_{1}(s), \ldots, x_{2 n}(s)$,

where

$$
\begin{aligned}
& x_{i}(s)=r_{i}(s) \hat{l}, \quad i=1, \ldots, 2 n, \quad \hat{l}=l /|l|, \\
& r_{2 j-1}(s)=\lambda_{j} s, \quad r_{2 j}(s)=\lambda_{j} s+\tau, \quad j=1, \ldots, n, \\
& \lambda_{1}=1, \quad \lambda_{j_{1}}<\lambda_{j_{2}} \text { for } j_{1}<j_{2}, \quad \tau>0 .
\end{aligned}
$$

This result is given in [N2] for finding $f_{1}$ from phaseless $\left|\psi^{+}\right|^{2}$, but can be also used for solving Problem 1.2.

Advantages of formulas (1.10), (1.11) (realized in the present work) in comparison with $(1.15),(1.16)$ (realized in [N2]) can be summarized as follows:

(i) for the same precision formulas (1.10), (1.11) use $n$ points instead of $2 n$;

(ii) the geometry of points in $(1.10),(1.11 \mathrm{a})$ is essencially simpler in the sense that the distances between points are fixed and are independent of $s \rightarrow+\infty$;

(iii) formulas (1.10), (1.11) (realized as formulas (1.14), (3.7), (3.11)) are drastically more explicit for large $n$. 
Results of the present work are presented in details in Section 3. These results (and in particular formulas $(1.14),(3.7),(3.11))$ also open question about simplification of formulas of [N2] for finfing $f_{1}$ from phaseless $\left|\psi^{+}\right|^{2}$.

\section{Preliminaries on direct scattering}

We recall that for equation (1.1), under assumptions (1.2), the scattering solutions $\psi^{+}$satisfy the Lippmann-Schwinger integral equation

$$
\begin{aligned}
& \psi^{+}(x, k)=e^{i k x}+\int_{D} G^{+}(x-y, k) v(y) \psi^{+}(y, k) d y, \\
& G^{+}(x, k) \stackrel{\text { def }}{=}-(2 \pi)^{-d} \int_{\mathbb{R}^{d}} \frac{e^{i \xi x} d \xi}{\xi^{2}-k^{2}-i 0}=G_{0}^{+}(|x|,|k|),
\end{aligned}
$$

where $x \in \mathbb{R}^{d}, k \in \mathbb{R}^{d}, k^{2}=E$. Note that

$$
\begin{aligned}
& G^{+}(x, k)=\frac{e^{i|k||x|}}{2 i|k|} \text { for } d=1, \\
& G^{+}(x, k)=-\frac{i}{4} H_{0}^{1}(|x||k|) \text { for } d=2, \quad G^{+}(x, k)=-\frac{e^{i|k||x|}}{4 \pi|x|} \text { for } d=3,
\end{aligned}
$$

where $H_{0}^{1}$ is the Hankel function of the first type.

Remark 2.1. In the present work, in addition to (1.2), we assume that

equation (2.1) is uniquely solvable for $\psi^{+}(\cdot, k) \in L^{\infty}\left(\mathbb{R}^{d}\right)$ for fixed $E>0$,

where $k \in \mathbb{R}^{d}, k^{2}=E$. If, for example, $v$ satisfies (1.2) and is real-valued, then (2.4) is fulfilled automatically.

In addition, for the normalized scattering amplitude $f$ defined by (1.6) the following formulas hold:

$$
\begin{gathered}
f(k, l)=(2 \pi)^{-d} \int_{D} e^{-i l y} v(y) \psi^{+}(y, k) d y \\
f(k, l)=(2 \pi)^{-d} \int_{\partial D}\left(e^{-i l x} \frac{\partial}{\partial \nu_{x}} \psi^{+}(x, k)-\psi^{+}(x, k) \frac{\partial}{\partial \nu_{x}} e^{-i l x}\right) d x,
\end{gathered}
$$

where $(k, l) \in \mathcal{M}_{E}, \nu_{x}$ is the outward normal to $\partial D$ at $x \in \partial D$.

For basic mathematical results concerning (2.1), (2.5) we refer to [F], [BSh], [CK], [N2], [N3] and references therein. Formula (2.6) follows from (2.5) using the Green formula; see formulas (4.1), (4.5) of [N1].

We also recall that, under our assumptions (1.2), (2.4), formula (1.4) admits the following much more precise version:

$$
\psi^{+}(x, k)=e^{i k x}+\frac{e^{i|k||x|}}{|x|^{(d-1) / 2}}\left(\sum_{j=1}^{n} \frac{f_{j}\left(k,|k| \frac{x}{|x|}\right)}{|x|^{j-1}}+O\left(\frac{1}{|x|^{n}}\right)\right) \text { as }|x| \rightarrow \infty,
$$


Multipoint formulas for scattered far field

where $x \in \mathbb{R}^{d}, k \in \mathbb{R}^{d}, k^{2}=E>0, n \in \mathbb{N}$. In addition, formulas (1.6), (1.7), (2.5) for $f_{1}$ have analogs for $f_{j}, j \geq 2$. See, for example, [M], [N2].

\section{Main results}

Let $z=z(s)$ be a function of $s \in[r,+\infty[, r>0$, such that

$$
z(s)=\sum_{j=1}^{N} \frac{f_{j}}{s^{j-1}}+O\left(s^{-N}\right) \text { as } s \rightarrow+\infty
$$

where $N \in \mathbb{N}, f_{j} \in \mathbb{C}, j=1, \ldots, N$.

Our considerations of functions satisfying (3.1) are motivated by formulas (1.12), (2.7). However, in this section we do not assume that $z=z(s)$ and $f_{1}, \ldots, f_{N}$ are related to equation (1.1) unless it is explicitly specified.

First, we consider $n$ points $s_{j} \in[r,+\infty[, j=1, \ldots, n$, of the form

$$
\begin{aligned}
& s_{j}=s_{j}(s)=s+\tau_{j}, \quad j=1, \ldots, n, \\
& s>r, \tau_{1}=0, \tau_{j_{1}}<\tau_{j_{2}} \text { for } j_{1}<j_{2} .
\end{aligned}
$$

We set

$$
\begin{gathered}
\vec{\tau}=\left(\tau_{1}, \ldots, \tau_{n}\right), \\
\alpha_{j}(\vec{\tau})=\prod_{i=1}^{j-1}\left(\tau_{j}-\tau_{i}\right) \text { for } 1<j \leq n, \quad \alpha_{1}(\vec{\tau})=1, \\
\beta_{n, j}(\vec{\tau})=\prod_{i=j+1}^{n}\left(\tau_{i}-\tau_{j}\right) \text { for } 1 \leq j<n, \quad \beta_{n, n}(\vec{\tau})=1 .
\end{gathered}
$$

In addition, if $\tau_{j}$ are given by (1.13), then we have that

$$
\alpha_{j}(\vec{\tau})=(j-1) ! \tau^{j-1}, \quad \beta_{n, j}(\vec{\tau})=(n-j) ! \tau^{n-j} .
$$

Theorem 3.1. Let $z=z(s)$ satisfy (3.1) for some $N \geq 2 n-1, n \in \mathbb{N}$. Let $\vec{\tau}$ be defined according to (3.2), (3.3). Then

$$
\begin{aligned}
& f_{1}=f_{1, n}(\vec{\tau}, s)+O\left(s^{-n}\right) \text { as } s \rightarrow+\infty \\
& f_{1, n}(\vec{\tau}, s)=\sum_{j=1}^{n} \frac{(-1)^{n-j}\left(s+\tau_{j}\right)^{n-1} z\left(s+\tau_{j}\right)}{\alpha_{j}(\vec{\tau}) \beta_{n, j}(\vec{\tau})}
\end{aligned}
$$

where $\alpha_{j}(\vec{\tau}), \beta_{n, j}(\vec{\tau})$ are defined by (3.4).

Theorem 3.1 is proved in Section 4.

Using Theorem 3.1 and formulas (1.12), (2.7) we obtain the following corollary. 
R.G. Novikov

Corollary 3.1. Under assumptions (1.2), (2.4), we have that

$$
\begin{aligned}
& f_{1}(k, l)=f_{1, n}(k, l, \vec{\tau}, s)+O\left(s^{-n}\right) \text { as } s \rightarrow+\infty, \\
& f_{1, n}(k, l, \vec{\tau}, s)=\sum_{j=1}^{n} \frac{(-1)^{n-j}\left(s+\tau_{j}\right)^{n-1} z\left(\left(s+\tau_{j}\right) \hat{l}, k\right)}{\alpha_{j}(\vec{\tau}) \beta_{n, j}(\vec{\tau})}, \\
& (k, l) \in \mathcal{M}_{E}, \hat{l}=l /|l|,
\end{aligned}
$$

where $f_{1}=f_{1}(k, l)$ is the scattering amplitude of (1.4), $z=z(x, k)$ is defined by (1.12), $\alpha_{j}(\vec{\tau}), \beta_{n, j}(\vec{\tau})$ are defined by (3.4), $\tau_{j}$ are the numbers of (1.11a).

Second, we also consider $n$ points $s_{j} \in[r,+\infty[, j=1, \ldots, n$, of the form

$$
\begin{aligned}
& s_{j}=s_{j}(s)=\lambda_{j} s, \quad j=1, \ldots, n, \\
& s>r, \quad \lambda_{1}=1, \quad \lambda_{j_{1}}<\lambda_{j_{2}} \text { for } j_{1}<j_{2} .
\end{aligned}
$$

Let

$$
\vec{\lambda}=\left(\lambda_{1}, \ldots, \lambda_{n}\right)
$$

Theorem 3.2. Let $z=z(s)$ satisfy (3.1) for some $N=n \in \mathbb{N}$. Let $\vec{\lambda}$ be defined according to (3.8), (3.9). Then

$$
\begin{aligned}
& f_{1}=f_{1, n}(\vec{\lambda}, s)+O\left(s^{-n}\right) \text { as } s \rightarrow+\infty, \\
& f_{1, n}(\vec{\lambda}, s)=\sum_{j=1}^{n} \frac{(-1)^{n-j} \lambda_{j}^{n-1} z\left(\lambda_{j} s\right)}{\alpha_{j}(\vec{\lambda}) \beta_{n, j}(\vec{\lambda})},
\end{aligned}
$$

where $\alpha_{j}(\vec{\lambda}), \beta_{n, j}(\vec{\lambda})$ are defined according to (3.4) (with $\vec{\lambda}$ in place of $\vec{\tau}$ ).

Theorem 3.2 is proved in Section 5 .

Using Theorem 3.2 and formulas (1.12), (2.7) we obtain the following corollary.

Corollary 3.2. Under assumptions (1.2), (2.4), we have that

$$
\begin{aligned}
& f_{1}(k, l)=f_{1, n}(k, l, \vec{\lambda}, s)+O\left(s^{-n}\right) \text { as } s \rightarrow+\infty, \\
& f_{1, n}(k, l, \vec{\lambda}, s)=\sum_{j=1}^{n} \frac{(-1)^{n-j} \lambda_{j}^{n-1} z\left(\lambda_{j} s \hat{l}, k\right)}{\alpha_{j}(\vec{\lambda}) \beta_{n, j}(\vec{\lambda})} \\
& (k, l) \in \mathcal{M}_{E}, \hat{l}=l /|l|,
\end{aligned}
$$

where $f_{1}=f_{1}(k, l)$ is the scattering amplitude of (1.4), $z=z(x, k)$ is defined by (1.12), $\alpha_{j}(\vec{\lambda}), \beta_{n, j}(\vec{\lambda})$ are defined according to (3.4) (with $\vec{\lambda}$ in place of $\vec{\tau}$ ), $\lambda_{j}$ are the numbers of (1.11b).

Remark 3.1. Formulas (3.6), (3.7), (3.10), (3.11) have also analogs for $f_{j}, j \geq 2$. For the case of (3.10), (3.11) these analogs are, actually, given by formulas (5.3), (5.9) of Section 5 . 
Multipoint formulas for scattered far field

\section{Proof of Theorem 3.1}

For $n=1$ formulas (3.6) are reduced to (3.1) for $N=1$.

Therefore, for simplicity of notations, further in this proof we assume that $n \geq 2$.

Let $y_{j}=y_{j}(s, \vec{\tau}), j=1, \ldots, n$, be defined via the following linear system

$$
\sum_{j=1}^{n} \frac{y_{j}}{\left(s+\tau_{j}\right)^{i-1}}=\left\{\begin{array}{l}
1 \text { for } i=1 \\
0 \text { for } 1<i \leq n .
\end{array}\right.
$$

System (4.1) can be rewritten as

$$
\begin{aligned}
& Q y=e_{1}, \\
& Q=\left(Q_{i, j}\right), \quad Q_{i, j}=q_{j}^{i-1}, \quad q_{j}=\left(s+\tau_{j}\right)^{-1}, \\
& y=\left(y_{1}, \ldots, y_{n}\right)^{T}, \quad e_{1}=(1,0, \ldots, 0)^{T} .
\end{aligned}
$$

One can see that $Q$ is a Vandermonde matrix and, in particular,

$$
\operatorname{det} Q=\prod_{1 \leq i<j \leq n}\left(q_{j}-q_{i}\right) \neq 0
$$

where we used our assumptions on $\tau_{j}$ of (3.2) in order to have that $\operatorname{det} Q \neq 0$.

Let

$$
z_{n}(s)=\sum_{j=1}^{n} \frac{f_{j}}{s^{j-1}} .
$$

Formulas (4.1), (4.4) imply that

$$
\sum_{j=1}^{n} y_{j}(s, \vec{\tau}) z_{n}\left(s+\tau_{j}\right)=f_{1} .
$$

In order to prove (3.6) it remains to show that:

$$
\begin{gathered}
y_{j}(s, \vec{\tau})=\frac{(-1)^{n-j}\left(s+\tau_{j}\right)^{n-1}}{\alpha_{j}(\vec{\tau}) \beta_{n, j}(\vec{\tau})}, \quad 1 \leq j \leq n, \\
\sum_{j=1}^{n} \frac{y_{j}(s, \vec{\tau})}{\left(s+\tau_{j}\right)^{i-1}}=O\left(s^{-n}\right) \text { as } s \rightarrow+\infty, \text { for } n<i<2 n .
\end{gathered}
$$

Indeed, formulas (3.6) follow from (3.1), $N \geq 2 n-1$, and (4.4)-(4.7).

Proof of (4.6). We recall that

$$
\begin{aligned}
Q_{i, j}^{-1} & =(-1)^{j-1}\left(\sum_{\substack{1 \leq m_{1}<\ldots<m_{n-j} \leq n \\
m_{1}, \ldots, m_{n-j} \neq i}} q_{m_{1}} \ldots q_{m_{n-j}}\right)\left(\prod_{\substack{1 \leq m \leq n \\
m \neq i}}\left(q_{m}-q_{i}\right)\right)^{-1}, 1 \leq j<n, \\
Q_{i, j}^{-1} & =(-1)^{j-1}\left(\prod_{\substack{1 \leq m \leq n \\
m \neq i}}\left(q_{m}-q_{i}\right)\right)^{-1}, j=n,
\end{aligned}
$$


where $Q^{-1}=\left(Q_{i, j}^{-1}\right)$ is the inverse of the Vandermonde matrix $Q$; see, e.g., Section 1.2.3: Sums and Products: Exercise 40 of $[\mathrm{K}]$.

Using (4.2), (4.8) we obtain that

$$
\begin{aligned}
& y_{i}(s, \vec{\tau})=\left(\sum_{\substack{1 \leq m_{1}<\ldots<m_{n-1} \leq n \\
m_{1}, \ldots, m_{n-1} \neq i}} q_{m_{1}} \ldots q_{m_{n-1}}\right)\left(\prod_{\substack{1 \leq m \leq n \\
m \neq i}}\left(q_{m}-q_{i}\right)\right)^{-1} \\
& =\left(\prod_{\substack{1 \leq m \leq n \\
m \neq i}} q_{m}\right)\left(\prod_{\substack{1 \leq m \leq n \\
m \neq i}}\left(q_{m}-q_{i}\right)\right)^{-1}, \quad 1 \leq i \leq n .
\end{aligned}
$$

Next, using the definition of $q_{j}$ in (4.2) we have that

$$
q_{m}-q_{i}=\left(\tau_{i}-\tau_{m}\right) q_{m} q_{i}
$$

Using (4.9), (4.10) and the definition of $q_{j}$ we obtain that

$$
y_{i}(s, \vec{\tau})=\frac{1}{q_{i}^{n-1} \prod_{\substack{1 \leq m \leq n \\ m \neq i}}\left(\tau_{i}-\tau_{m}\right)}=\frac{\left(s+\tau_{i}\right)^{n-1}}{\prod_{\substack{1 \leq m \leq n \\ m \neq i}}\left(\tau_{i}-\tau_{m}\right)}, \quad 1 \leq i \leq n
$$

Formulas (4.11), (3.4) imply (4.6).

Proof of (4.7). Using (4.6) we obtain that

$$
\sum_{j=1}^{n} \frac{y_{j}(s, \vec{\tau})}{\left(s+\tau_{j}\right)^{i-1}}=\sum_{j=1}^{n} \frac{\gamma_{n, j}(\vec{\tau})}{\left(s+\tau_{j}\right)^{i-n}}=\frac{1}{s^{i-n}} \sum_{j=1}^{n} \gamma_{n, j}(\vec{\tau})\left(1+\frac{\tau_{j}}{s}\right)^{-(i-n)},
$$

where $i>n, s>0$,

$$
\gamma_{n, j}(\vec{\tau})=\frac{(-1)^{n-j}}{\alpha_{j}(\vec{\tau}) \beta_{n, j}(\vec{\tau})}
$$

We recall that

$$
\left(1+\frac{\tau_{j}}{s}\right)^{-(i-n)}=\sum_{k=0}^{+\infty} C_{n-i}^{k}\left(\frac{\tau_{j}}{s}\right)^{k} \text { as } s \rightarrow+\infty
$$

where $C_{n-i}^{k}$ are the binomial coefficients.

We also assume that $\tau_{1}^{0}=1$ (in view of our assumption that $\tau_{1}=0$ ).

Using (4.14) we obtain that

$$
\sum_{j=1}^{n} \gamma_{n, j}(\vec{\tau})\left(1+\frac{\tau_{j}}{s}\right)^{-(i-n)}=\sum_{k=0}^{n-2} C_{n-i}^{k} \sigma_{n, k}(\vec{\tau}) \frac{1}{s^{k}}+O\left(\frac{1}{s^{n-1}}\right), \quad s \rightarrow+\infty
$$


Multipoint formulas for scattered far field

where

$$
\sigma_{n, k}(\vec{\tau})=\sum_{j=1}^{n} \gamma_{n, j}(\vec{\tau}) \tau_{j}^{k}
$$

Because of (4.12), (4.15), in order to complete the proof of (4.7) it is sufficient to show that

$$
\sigma_{n, k}(\vec{\tau})=0, \quad k=0, \ldots, n-2 .
$$

On this purpose, using (4.1) for $i=1$ and (4.6), (4.13), (4.16) we obtain that

$$
\begin{aligned}
& \sum_{j=1}^{n} y_{j}(s, \vec{\tau})=\sum_{j=1}^{n} \gamma_{n, j}(\vec{\tau})\left(s+\tau_{j}\right)^{n-1}=\sum_{j=1}^{n} \gamma_{n, j}(\vec{\tau}) \sum_{k=0}^{n-1} C_{n-1}^{k} s^{n-1-k} \tau_{j}^{k} \\
& =\sum_{k=0}^{n-1} C_{n-1}^{k} s^{n-1-k} \sigma_{n, k}(\vec{\tau})=1, \quad s>0
\end{aligned}
$$

where $C_{n-1}^{k}$ are the binomial coefficients.

The identities (4.17) follow from (4.18).

This completes the proof of formulas (4.7).

Theorem 3.1 is proved.

\section{Proof of Theorem 3.2}

We set

$$
\Lambda=\left(\Lambda_{j_{1}, j_{2}}\right)=\left(\lambda_{j_{1}}^{1-j_{2}}\right), \quad j_{1}, j_{2}=1, \ldots, n,
$$

where $\lambda_{1}, \ldots, \lambda_{n}$ are the numbers of (3.8). One can see that $\Lambda$ is a Vandermonde matrix and, in particular,

$$
\operatorname{det} \Lambda=\prod_{1 \leq i<j \leq n}\left(\lambda_{j}^{-1}-\lambda_{i}^{-1}\right) \neq 0 \text { for } n \geq 2, \quad \operatorname{det} \Lambda=\lambda_{1}=1 \text { for } n=1 \text {. }
$$

Proposition 5.1. Under the assumptions of Theorem 3.2, the following formulas hold:

$$
\begin{aligned}
& f_{i}=f_{i, n-i+1}(\vec{\lambda}, s)+O\left(s^{-n+i-1}\right) \text { as } s \rightarrow+\infty, \\
& f_{i, n-i+1}(\vec{\lambda}, s)=s^{i-1} \sum_{j=1}^{n} \Lambda_{i, j}^{-1} z\left(\lambda_{j} s\right), \quad i=1, \ldots, n,
\end{aligned}
$$

where $\Lambda^{-1}=\left(\Lambda_{i, j}^{-1}\right)$ is the inverse of the matrix $\Lambda$ defined by (5.1).

Proof of Proposition 5.1. Proceeding from (3.1) we obtain the following system of approximate equations for $f_{1}, \ldots, f_{n}$ :

$$
\sum_{j=1}^{n} \frac{f_{j}}{\left(\lambda_{i} s\right)^{j-1}}=z\left(\lambda_{i} s\right)+O\left(s^{-n}\right) \text { as } s \rightarrow+\infty, \quad i=1, \ldots, n \text {. }
$$


R.G. Novikov

Let

$$
\begin{aligned}
& g(s)=\left(g_{1}(s), \ldots, g_{n}(s)\right)^{T}, \quad g_{j}(s)=\frac{f_{j}}{s^{j-1}}, \quad j=1, \ldots, n, \\
& h(s)=\left(h_{1}(s), \ldots, h_{n}(s)\right)^{T}, \quad h_{j}(s)=z\left(\lambda_{j} s\right), j=1, \ldots, n .
\end{aligned}
$$

Then system (5.4) can be rewritten as

$$
\Lambda g(s)=h(s)+O\left(s^{-n}\right) \text { as } s \rightarrow+\infty
$$

where $O\left(s^{-n}\right)$ denotes an $n$-dimensional vector with the components $O\left(s^{-n}\right)$.

From (5.2), (5.7) we obtain that

$$
g(s)=\Lambda^{-1} h(s)+O\left(s^{-n}\right) \text { as } s \rightarrow+\infty .
$$

Formulas (5.3) follow from (5.5), (5.6), (5.8).

Proposition 5.1 is proved.

The rest of the proof of Theorem 3.2 is as follows.

For $n=1$ formulas (3.10) coincide with (3.6) and with (5.3) and are reduced to (3.1) for $N=1$.

Therefore, for simplicity of notations, further in this proof we assume that $n \geq 2$.

In a similar way with (4.8) we have that

$$
\begin{aligned}
& \Lambda_{i, j}^{-1}=(-1)^{i-1}\left(\sum_{\substack{1 \leq m_{1}<\ldots<m_{n-i} \leq n \\
m_{1}, \ldots, m_{n-i} \neq j}} \lambda_{m_{1}}^{-1} \ldots \lambda_{m_{n-i}}^{-1}\right)\left(\prod_{\substack{1 \leq m \leq n \\
m \neq j}}\left(\lambda_{m}^{-1}-\lambda_{j}^{-1}\right)\right)^{-1}, 1 \leq i<n, \\
& \Lambda_{i, j}^{-1}=(-1)^{i-1}\left(\prod_{\substack{1 \leq m \leq n \\
m \neq j}}\left(\lambda_{m}^{-1}-\lambda_{j}^{-1}\right)\right)^{-1}, i=n .
\end{aligned}
$$

Using (5.9), in a similar way with (4.9), (4.10), (4.11) we have that

$$
\begin{gathered}
\Lambda_{1, j}^{-1}=\left(\prod_{\substack{1 \leq m \leq n \\
m \neq j}} \lambda_{m}^{-1}\right)\left(\prod_{\substack{1 \leq m \leq n \\
m \neq j}}\left(\lambda_{m}^{-1}-\lambda_{j}^{-1}\right)\right)^{-1}, \quad 1 \leq j \leq n, \\
\lambda_{m}^{-1}-\lambda_{j}^{-1}=\left(\lambda_{j}-\lambda_{m}\right) \lambda_{m}^{-1} \lambda_{j}^{-1}, \\
\Lambda_{1, j}^{-1}=\frac{\lambda_{j}^{n-1}}{\prod_{\substack{1 \leq m \leq n \\
m \neq j}}\left(\lambda_{j}-\lambda_{m}\right)}, \quad 1 \leq j \leq n .
\end{gathered}
$$

Formulas (3.10) follow from formulas (5.3) for $i=1$ and formulas (5.12). Theorem 3.2 is proved. 
Multipoint formulas for scattered far field

\section{References}

[Ber] Yu.M. Berezanskii, On the uniqueness theorem in the inverse problem of spectral analysis for the Schrödinger equation, Tr. Mosk. Mat. Obshch. 7, 3-62 (1958) (in Russian); English transl.: Am. Math. Soc. Trans. 35, 167-235 (1964)

[BSh] F.A. Berezin, M.A. Shubin, The Schrödinger Equation, Vol. 66 of Mathematics and Its Applications, Kluwer Academic, Dordrecht, 1991.

[ CK] D. Colton R. Kress, Inverse Acoustic and Electromagnetic Scattering Theory, 4th. ed. Applied Mathematical Sciences, 93. Springer, Cham, 2019.

[F] L.D. Faddeev, Inverse problem of quantum scattering theory. II, Itogy Nauki i Tekh. Ser. Sovrem. Probl. Mat. 3, 93-180 (1974) (in Russian); English transl.: Journal of Soviet Mathematics 5, 334-396 (1976)

[ K] D.E. Knuth, The Art of Computer Programming: Volume 1: Fundamental Algorithms (3rd Edition). Addison-Wesley, 1997.

[ M] R.B. Melrose, Geometric Scattering Theory. Stanford Lectures. Cambridge University Press, 1995.

[ N1] R.G. Novikov, Multidimensional inverse spectral problem for the equation $-\Delta \psi+$ $(v(x)-E u(x)) \psi=0$, Funkt. Anal. Prilozhen. 22(4), 11-22 (1988) (in Russian); English transl.: Funct. Anal. Appl. 22, 263-272 (1988)

[ N2] R.G. Novikov, Multipoint formulas for phase recovering from phaseless scattering data, J. Geom. Anal., doi:10.1007/s12220-019-00329-6

[ N3] R.G. Novikov, Multidimensional inverse scattering for the Schrödinger equation, epreprint: https://hal.archives-ouvertes.fr/hal-02465839v1 